\title{
Nitrogen assisted etching of graphene layers in a scanning electron microscope
}

\author{
D. Fox, ${ }^{1,2}$ A. O'Neill, ${ }^{1}$ D. Zhou, ${ }^{1,2}$ M. Boese, ${ }^{2}$ J. N. Coleman, ${ }^{1}$ and H. Z. Zhang ${ }^{1,2, a)}$ \\ ${ }^{1}$ School of Physics and CRANN, Trinity College Dublin, Dublin 2, Ireland \\ ${ }^{2}$ CRANN Advanced Microscopy Laboratory, Trinity College Dublin, Dublin 2, Ireland
}

(Received 10 May 2011; accepted 27 May 2011; published online 17 June 2011)

\begin{abstract}
We describe the controlled patterning of nanopores in graphene layers by using the low-energy $(<10 \mathrm{keV})$ focused electron beam in a scanning electron microscope. Regular nanometer-sized holes can be fabricated with the presence of nitrogen gas. The effect of the gas pressure, beam current, and energy on the etching process are investigated. Transmission electron microscopy, coupled with plasmon energy loss imaging, reveals the microstructure modification of the etched graphene. A nitrogen-ion assisted etching mechanism is proposed for the controlled patterning.

(C) 2011 American Institute of Physics. [doi:10.1063/1.3601467]
\end{abstract}

Graphene, a two-dimensional hexagonal lattice of $s p^{2}$ bonded carbon atoms, has received a huge amount of interest since its isolation in 2004. ${ }^{1}$ It exhibits unique properties such as ballistic transport of charge carriers, ${ }^{2}$ extremely high thermal conductivity, ${ }^{3}$ and the highest breaking strength. ${ }^{4}$ However, for device applications it is necessary to tailor the properties of this material. Take, for example, graphene nanoribbons which are engineered to have dimensions less than $10 \mathrm{~nm}$. The edges of the ribbons have localized states which affect transport behaviors, this allows the introduction of a controllable band-gap in graphene. ${ }^{5}$ The addition of defects can also lead to a modified band structure and resulted in enhanced conductivity. ${ }^{6}$ Porous graphene surfaces have potential applications in nanofiltration, ${ }^{7}$ DNA sequencing, ${ }^{8}$ and hydrogen storage. ${ }^{9}$

Many methods have been used to achieve precise control of graphene's properties, both chemically ${ }^{10-12}$ and physically; ${ }^{13-17}$ however, they suffer from limitations such as the introduction of performance inhibiting contaminants or the use of specialized equipment. The scanning electron microscope (SEM) is a widely available surface characterization tool which is well established for nanofabrication in today's fabrication facilities. In this work, we develop an SEM-based technique to modify the geometry of graphene samples at the nanoscale and image the modification in situ. The mechanism of the modification was studied systematically.

The graphene sample was prepared by sonicating pristine graphite in isopropanol for $48 \mathrm{~h}$. After sonication, the sample was centrifuged at $2000 \mathrm{rpm}$ for $45 \mathrm{~min}$ to remove large chucks of graphite that did not fully exfoliate. The resulting supernatant was decanted and retained for further use. The dispersion quality was similar to that previously reported by O'Neill et al., ${ }^{18}$ with flakes typically $1 \mu \mathrm{m}$ in length and with thicknesses of less than ten layers. The dispersion was dropped onto a lacey carbon transmission electron microscopy (TEM) grid and allowed to dry naturally in air. All SEM was performed using a Carl Zeiss Ultra Plus SEM. In each etching experiment the electron beam was focused onto a spot on the surface of the graphene flake. A nitrogen flux was delivered directly to the adjacent region of

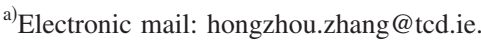

the beam-sample interaction spot through a nozzle. The flux was adjusted by monitoring the overall vacuum of the SEM chamber. Etching of the graphene was studied as a function of beam dwell time, nitrogen gas pressure, beam current, and beam energy. The amount of material etched was measured to extract the etching rate. An FEI Titan TEM operating at $80 \mathrm{kV}$ was used to analyze the effect of the etching process on our sample.

Etching of the graphene flakes was found to occur when the electron beam was focused in the presence of nitrogen. Holes of several tens of nanometer in diameter were introduced to the graphene flake as shown in Fig. 1(a). Figure 1(b) shows a plasmon filtered energy loss image of the edge morphology of a typical hole. The contrast in the image indicates thickness variation with monolayer precision. ${ }^{19}$ It is

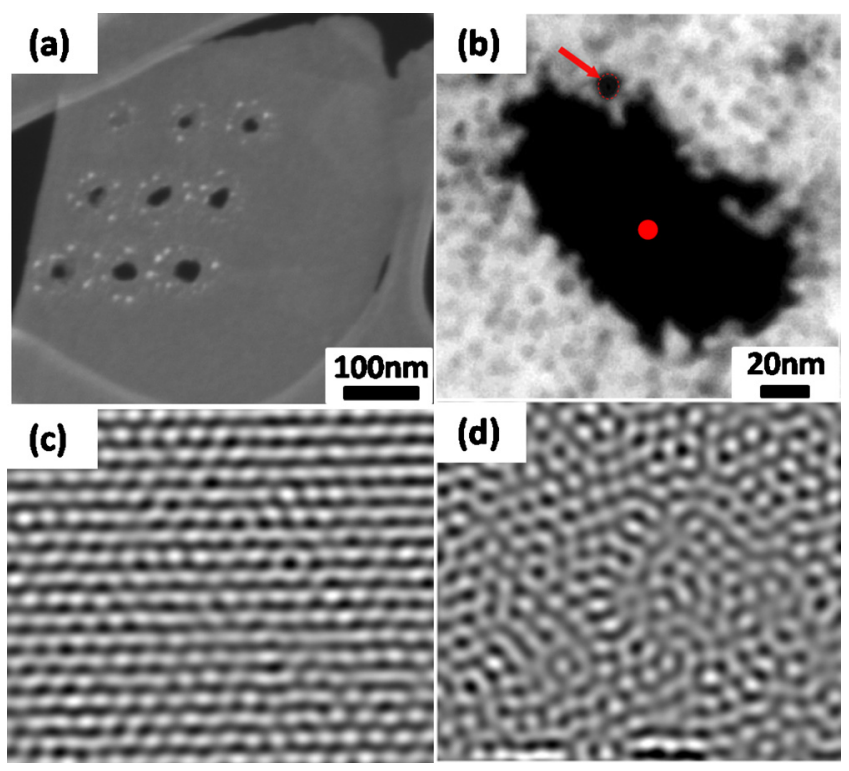

FIG. 1. (Color online) (a) SEM image of nine holes etched in a graphene flake. (b) Plasmon energy loss image with material thickness contrast, the dot at the hole center shows the nominal diameter of the SEM electron beam used to etch the hole $(5.9 \mathrm{~nm} \pm 0.4 \mathrm{~nm}$ ). (c) A focal series reconstruction of a pristine graphene lattice. (d) A focal series reconstruction of graphene adjacent to a region etched in the SEM. The etching process is observed to introduce defects into the lattice when compared with the lattice before etching in (c). 


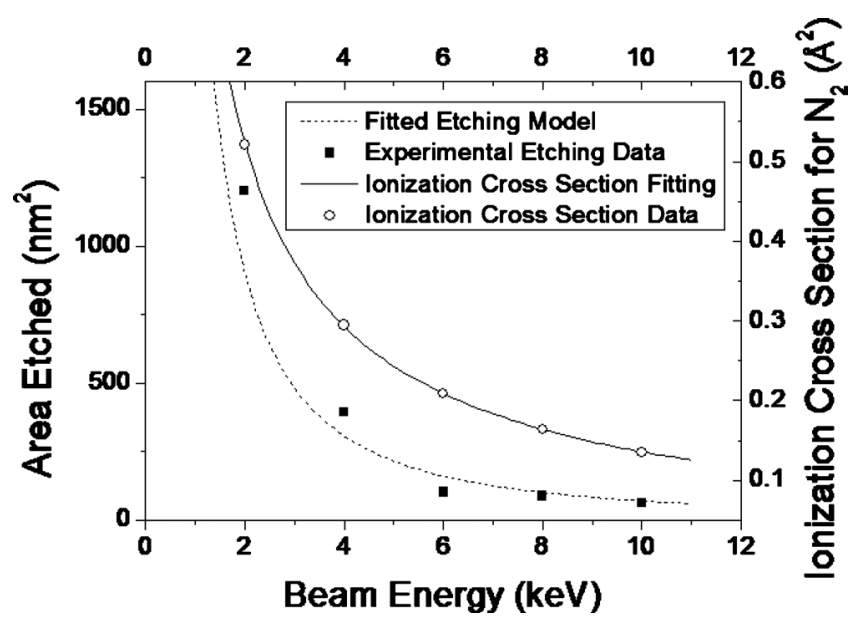

FIG. 2. A logarithmic increase in the calculated ionization cross section of nitrogen gas occurs as the energy of the electron beam is reduced (open circles with solid fitted line). The dashed curve is a fitting of our model from Eq. (3). The black squares represent the actual data recorded during the experiment.

evident that nanosized pores $(<10 \mathrm{~nm}$ in diameter), indicated by the arrow in Fig. 1(b), are distributed in a region that extends about $80 \mathrm{~nm}$ from the hole edge. The nominal size of the SEM probe is also shown in Fig. 1(b) (red dot at the center). The etching effect is observed to extend well beyond the region of the focused beam of the SEM.

Lattice-resolved TEM images of pristine and etched graphene flakes are shown in Figs. 1(c) and 1(d), respectively. Focal serial reconstruction has been conducted to achieve the information limit. A high pass filter has been applied to the reconstructed images, this allows us to highlight the high frequency information and remove the "foggy" background. The bright spots in our image correspond to positions at which the atoms in each layer overlap. The lattice of the pristine graphene flake in Fig. 1(c) is ordered and shows hexagonal symmetry. The etched flake's [Fig. 1(d)] lattice structure has clearly been degraded during the etching process leading to the introduction of lattice damage and amorphization.

To study the etching mechanism, several experiments were conducted to observe the effect of varying a range of parameters in the system. The time of exposure to the beam, the pressure of the nitrogen gas in the system, and the beam current were each varied over a range of values individually. The area etched was found to increase linearly with increasing beam dwell time. It was also found that an increase in the gas pressure, or the beam current, resulted in a linear increase in the etching rate. It is apparent from the experimental data in Fig. 2 (the black squares) that the etching rate is strongly dependant on the beam energy, a strong increase in etching rate is observed for lower energy electrons.

The etching that we have observed experimentally can be described by the following model. As illustrated in Fig. 3, the first process occurring is the ionization of the nitrogen gas due to the electron beam. When these chemically active nitrogen ions are generated in the region of the graphene surface a reaction occurs. A product of the reaction between activated nitrogen and graphite surfaces has been found to be cyanogen $^{20}(\mathrm{CN})_{2}$, a gaseous compound. The reaction product is removed from the surface and pumped out of the SEM chamber. This leads to the removal of material from the

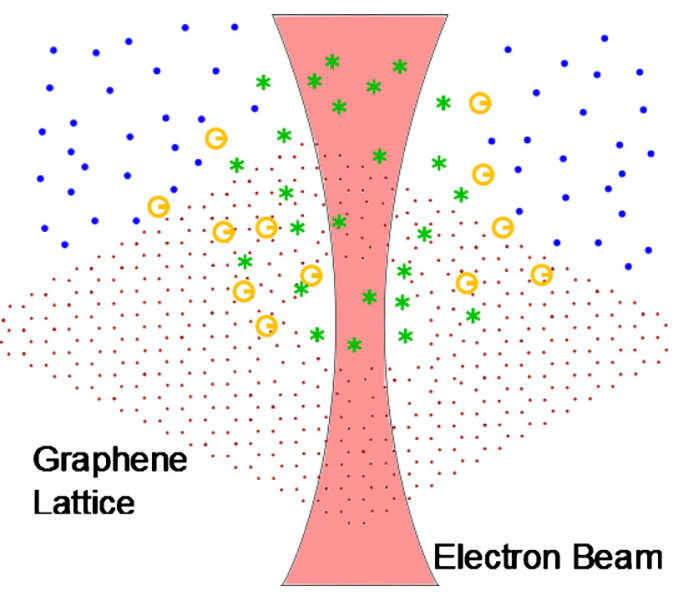

FIG. 3. (Color online) An illustration of the etching process. The nitrogen gas (blue circles) is ionized (green asterisks) by the electron beam (pink cone at the center). The nitrogen ions bond with carbon atoms to form a gaseous product (hollow circles) which is then pumped from the system.

sample by localized reactive-ion etching, a process widely used in fabrication facilities. ${ }^{21}$ Once a small pore is formed its edges are the preferred sites for bonding with nitrogen ions, pores are therefore observed to grow radially on the irradiated graphene surface.

The increased etching due to both the longer beam dwell time and the higher nitrogen pressure can then be considered to be due simply to the greater number of nitrogen ions generated. A greater number of ions will result in more reactions and therefore more graphene etching occurs. The variation in etching rate as a function of beam energy is due to the energy dependence of beam-nitrogen and beam-graphene interactions since the activation energy required for this chemical reaction is supplied by the electron beam to both the sample, thermally, and also to the gas, directly by ionization. The etching rate is therefore proportional to the energy transferred from the electron beam to both the nitrogen gas and the graphene sample.

The effect of the beam energy on the etching rate can be understood quantitatively (see Fig. 2) by considering the ionization cross sections. The number of nitrogen ions generated by the electron beam is proportional to the ionization cross section, $\sigma$, of nitrogen, which can be described by the binary-encounter-Bethe ${ }^{22}$ model. The cross sections of nitrogen were calculated for the energy range used in the experiments and are shown as open circles in Fig. 2. A log fitting of the modeled data was then used to get a simple analytical form (black solid line in Fig. 2) to account for the contribution to the change in etching rate due to the variation in the probability of ionization of nitrogen with beam energy,

$$
R=k^{\prime} \exp (-0.066) E_{i}^{(-0.84)} .
$$

It can be seen that in terms of the electron beam energy dependence, the observed etching behavior (solid square data points) deviates qualitatively from the nitrogen ionization. It indicates the etching process is also regulated by other physical processes.

As the electron beam penetrates the graphene layer, it deposits a certain amount of energy to the graphene lattice and that energy facilitates the chemical reaction which is responsible for the material removal. We assume the etching 
rate is proportional to the energy loss of the electron beam in the graphene layer, ${ }^{23} d E / d s$,

$$
R=k^{\prime \prime} \frac{d E}{d s}=-k^{\prime \prime} \frac{8.2}{E_{i}} \ln \left[12 E_{i}\left(1+\frac{0.078}{E_{i}}\right)\right],
$$

where $E_{i}$ is the energy of the incident electron beam. Here, we consider only the surface since graphene is atomically thin. The beam energy dependence of the etching rate is therefore given by the two independent processes that occur in sequence, and by combining Eqs. (1) and (2) the etching rate as a function of the beam energy is given by

$$
R=k \exp (-0.066) E_{i}^{(-0.84)} \frac{8.2}{E_{i}} \ln \left[12 E_{i}\left(1+\frac{0.078}{E_{i}}\right)\right] .
$$

The only fitting parameter is the proportionality constant $k$, which represents a one-dimensional etching rate per thousand electron volt of energy of the electron beam. This model is used with $k=1.32 \mathrm{~nm} \mathrm{~s}^{-1} \mathrm{keV}^{-1}$ to plot the dashed curve in Fig. 2. The curve is in good agreement with our experimental data in Fig. 2 (black squares). This result indicates that the etching rate is dominated by the probabilities of nitrogen ionization and beam-graphene interaction in our system and the combined effect of these two processes determines the etching rate observed experimentally.

Our experimental and theoretical analyses corroborate the chemical etching model. Nitrogen ions react with the activated graphene surface to form a gaseous product and carbon atoms are removed initially. The vacancies which these leave behind cause the neighboring atoms to rearrange due to surface tension [see Fig. 1(d)]. The graphene lattice undergoes structural disorder and amorphization by these processes, leading to the worst affected areas growing into the pores which we observed in Fig. 1(b). A hole was formed where the electron beam was centered due to a large density of these pores. Nitrogen ions diffuse from the region of the electron beam and damage an area much larger than the beam spot size. It is worth noting that the experimental etching rate is slightly higher than the model predicated at lower beam energies. The lower energy electrons interact far more strongly with other atoms in the system. ${ }^{24}$ The high density of nanopores and their connection result in removal of large areas of graphene at an enhanced rate.

We have demonstrated controllable nanopore formation in graphene with a low energy electron beam. Nanopores with a diameter of $<10 \mathrm{~nm}$ have been fabricated. This method can be scaled up to industrial applications due to the ubiquity and relative inexpensiveness of low energy electron microscopes. The model proposed here relies on the generation of nitrogen ions but other gasses may also be used. Such modifications can have applications in gas filtration, ${ }^{7}$ DNA sequencing, ${ }^{8}$ and can even increase the carrier density of graphene.
We would like to acknowledge Dr. Gavin Behan and Mr. Cathal McAuley at the CRANN Advanced Microscopy Laboratory in Trinity College for their assistance and useful discussions. The work at the School of Physics and the Centre for Research on Adaptive Nanostructures and Nanodevices at Trinity College Dublin is supported by Science Foundation Ireland under Grant No. 07/SK/I1220a. The TEM work was conducted under the framework of the INSPIRE program, funded by the Irish Government's Program for Research in Third Level Institutions, Cycle 4, National Development Plan 2007-2013. A.O'N. would also like to acknowledge IRCSET for financial support.

${ }^{1}$ K. Novoselov, A. Geim, S. Morozov, D. Jiang, Y. Zhang, S. Dubonos, I. Grigorieva, and A. Firsov, Science 306, 666 (2004).

${ }^{2}$ X. Du, I. Skachko, A. Barker, and E. Andrei, Nat. Nanotechnol. 3, 491 (2008).

${ }^{3}$ A. A. Balandin, S. Ghosh, W. Z. Bao, I. Calizo, D. Teweldebrhan, F. Miao, and C. N. Lau, Nano Lett. 8, 902 (2008).

${ }^{4}$ C. Lee, X. Wei, J. Kysar, and J. Hone, Science 321, 385 (2008).

${ }^{5}$ L. Tapasztó, G. Dobrik, P. Lambin, and L. P. Biró, Nat Nanotechnol. 3, 397 (2008).

${ }^{6}$ S. H. M. Jafri, K. Carva, E. Widenkvist, T. Blom, B. Sanyal, J. Fransson, O. Eriksson, U. Jansson, H. Grennberg, O. Karis, R. A. Quinlan, B. C. Holloway, and K. Leifer, J. Phys. D: Appl. Phys. 43, 045404 (2010).

${ }^{7}$ S. Blankenburg, M. Bieri, R. Fasel, K. Müllen, C. A. Pignedoli, and D. Passerone, Small 6, 2266 (2010).

${ }^{8}$ H. Postma, Nano Lett. 10, 420 (2010).

${ }^{9}$ P. Reunchan and S.-H. Jhi, Appl. Phys. Lett. 98, 093103 (2011).

${ }^{10}$ O. Leenaerts, B. Partoens, and F. M. Peeters, Phys. Rev. B 77, 125416 (2008)

${ }^{11}$ H. E. Romero, N. Shen, P. Joshi, H. R. Gutierrez, S. A. Tadigadapa, J. O. Sofo, and P. C. Eklund, ACS Nano 2, 2037 (2008).

${ }^{12}$ V. A. Coleman, R. Knut, O. Karis, H. Grennberg, U. Jansson, R. Quinlan, B. C. Holloway, B. Sanyal, and O. Eriksson, J. Phys. D: Appl. Phys. 41, 062001 (2008).

${ }^{13}$ M. D. Fischbein and M. Drndic, Appl. Phys. Lett. 93, 113107 (2008).

${ }^{14}$ G. Dobrik, L. Tapasztó, P. Nemes-Incze, P. Lambin, and L. P. Biró, Phys. Status Solidi B 247, 896 (2010).

${ }^{15}$ M. C. Lemme, D. C. Bell, J. R. Williams, L. A. Stern, B. W. H. Baugher, P. Jarillo-Herrero, and C. M. Marcus, ACS Nano 3, 2674 (2009).

${ }^{16}$ L. Liu, S. M. Ryu, M. R. Tomasik, E. Stolyarova, N. Jung, M. S. Hybertsen, M. L. Steigerwald, L. E. Brus, and G. W. Flynn, Nano Lett. 8, 1965 (2008).

${ }^{17}$ P. S. Spinney, D. G. Howitt, R. L. Smith, and S. D. Collins, Nanotechnology 21, 375301 (2010).

${ }^{18}$ A. O'Neill, U. Khan, P. N. Nirmalraj, J. Boland, and J. N. Coleman, J. Phys. Chem. C 115, 5422 (2011).

${ }^{19}$ M. Boese, S. Kumar, A. O'Neill, M. Lotya, H. Zhang, J. Coleman, and G. Duesberg, Microsc. Microanal. 16, 1540 (2010).

${ }^{20}$ H. W. Goldstein, J. Phys. Chem. 68, 39 (1964).

${ }^{21}$ H. Abe, M. Yoneda, and N. Fujiwara, Jpn. J. Appl. Phys. 47, 1435 (2008).

${ }^{22}$ Y.-K. Kim and M. E. Rudd, Phys. Rev. A 50, 3954 (1994).

${ }^{23}$ J. Goldstein, D. E. Newbury, D. C. Joy, C. E. Lyman, P. Echlin, E. Lifshin, L. Sawyer, and J. R. Michael, Scanning Electron Microscopy and X-Ray Microanalysis (Springer, New York, 2003), Vol. 1, pp. 63-65.

${ }^{24}$ A. Kadoun, R. Belkorissat, B. Khelifa, and C. Mathieu, Vacuum 69, 537 (2003) 